\title{
Simulations of beam-beam effect in the presence of general chromaticity
}

\author{
D. Zhou, K. Ohmi, Y. Seimiya, Y. Ohnishi, A. Morita, and H. Koiso \\ KEK/SOKENDAI, 1-1 Oho, Tsukuba 305-0801, Japan \\ (Received 5 November 2009; published 3 February 2010)
}

\begin{abstract}
Optics parameters such as betatron tunes, beta functions, and dispersions depend on momentum deviation. Such dependence is characterized by general chromaticity. General chromaticity can induce emittance growth and luminosity degradation in colliders. To investigate the influence of general chromaticity on beam dynamics, a symplectic map for chromaticity was implemented in the beambeam codes and used in simulations at KEKB. A systematic investigation revealed that chromatic $X-Y$ couplings can deteriorate the machine luminosity in the order of $10 \%$ with head-on colliding beams. The results of this study triggered the installation of skew sextupoles to KEKB rings for the purpose of correcting the chromatic $X-Y$ couplings. Beam commissioning with skew-sextupole tuning knobs at KEKB has proved to be very successful; a new world's record luminosity of $2.1 \times 10^{34} \mathrm{~cm}^{-2} \mathrm{~s}^{-1}$ was achieved with Belle taking data in June, 2009. In this paper, we discuss the approach of general chromaticity and present the results of simulations at KEKB.
\end{abstract}

DOI: 10.1103/PhysRevSTAB.13.021001

PACS numbers: 29.20.db, 29.27.Bd

\section{INTRODUCTION}

The commissioning of KEKB [1] with crab cavities began in February 2007. Beam-beam simulations showed that the crab crossing is expected to boost the luminosity by a factor of 2 [2]. However, the luminosity is still lower than that predicted at high beam currents. Some research has investigated several candidates for sources of luminosity degradation at KEKB [3-5]. As pointed out in Ref. [3], lattice nonlinearities, including amplitude-dependent and momentum-dependent nonlinearities, may be important candidates.

The ultimate luminosity performance of a collider can be affected by a crosstalk between beam-beam interaction and lattice nonlinearities. For example, a relevant impact of the cubic nonlinearities on the peak luminosity and beam lifetime has been observed at the lepton colliders VEPP4M [6] and DAФNE [7]. A detailed simulation of beam-beam interaction and full treatment of lattice nonlinearities suggested a significant loss of luminosity in CESR-c that comes from momentum-dependent coupling errors in the detector solenoid compensation scheme [8]. These studies resulted in relevant interaction region modifications and luminosity improvement in CESR-c [9]. Recently, the influence of beta-function chromaticity at the IP has been studied at the Tevatron [10]. It was shown that large chromatic perturbations can lead to a decrease of the dynamic aperture for off-momentum particles due to head-on collisions and cause deterioration of the proton lifetime.

Recently, Seimiya and Ohmi devised a symplectic formalism to treat the momentum-dependent nonlinearities in a storage ring [11]. In their framework, they generalized the concept of chromaticity (so-called general chromaticity) as being characteristics of all optics parameters. That is, optics parameters such as betatron tunes, beta functions, and $X-Y$ couplings are functions of momentum deviation. Here we use "general chromaticity" to differentiate the traditional concept of "chromaticity," which usually has the meaning of chromatic dependence of betatron tunes. One can define general chromaticity as the coefficients of optics parameters expanding in a series of momentum deviation. General chromaticity was used to construct a symplectic map as perturbations in both betatron and synchrotron motions. This provided the possibility of simulating the beam-beam effect combined with momentumdependent nonlinearities in storage-ring colliders.

General chromaticity is an essential property of a machine and plays an important role in the beam dynamics, affecting machine properties such as the dynamic aperture, the beam lifetime, and the luminosity. The effect of normal tune chromaticity on beam dynamics has been studied extensively. There are some reports on treatments of the chromaticity of other optics parameters [12]. However, our study is an attempt to place general chromaticity in beambeam simulations in a general framework. Though we consider the KEKB as an example, we attempt to describe the theories and simulation techniques in a universal way.

General chromaticity can be measured by performing beam experiments in a well-tuned storage ring or calculated by using models with or without machine errors in beam optics codes such as SAD [13]. Though machine errors can be important sources of additional general chromaticity, their contributions will not be emphasized here, other than short discussions in the context of this study.

The rest of this paper is organized as follows. First, the symplectic formalism for general chromaticity is briefly reviewed in Sec. II. The implementation of the formalism in the simulation codes is presented in Sec. III. In Sec. IV, the first measurement of general chromaticity at the KEKB 
HER, with a comparison with SAD modeling is presented. In Secs. V, VI, and VII, the beam-beam effect with general chromaticity is discussed by using KEKB as an example. A summary of the results is presented in Sec. VIII.

The reader must note that, in the context of this paper, we are always referring to general chromaticity besides some special cases such as tune chromaticity. Further, we will abbreviate general chromaticity as chromaticity. The investigation of chromaticity of dispersion functions was beyond the scope of this study, though they have already been included in the chromaticity formalism [11].

\section{GENERALIZED CONCEPT OF CHROMATICITY REVISITED}

In the literature, chromaticity has mostly been considered to be a result of a betatron tune shift due to momentum deviation. However, generally speaking, all optics parameters vary with momentum deviation. The generalized concept of chromaticity originates from such chromatic characteristics of betatron motion. In this paper, we discuss the chromaticities of Twiss parameters and $X-Y$ couplings, which are expressed as a power series with respect to relative momentum deviation $\delta=\left(p-p_{0}\right) / p_{0}$ as follows:

$$
\begin{array}{rlrl}
\alpha_{u}(\delta) & =\sum_{i=0}^{\infty} \alpha_{u i} \delta^{i} & \beta_{u}(\delta)=\sum_{i=0}^{\infty} \beta_{u i} \delta^{i} \\
\nu_{u}(\delta) & =\sum_{i=0}^{\infty} \nu_{u i} \delta^{i} & r_{j}(\delta)=\sum_{i=0}^{\infty} r_{j i} \delta^{i} \\
u & =x, y \quad \text { and } \quad j=1,2,3,4,
\end{array}
$$

where the zero-order terms $\alpha_{u 0}, \beta_{u 0}, \nu_{u 0}$, and $r_{j 0}$ are the on-momentum alpha function, beta function, betatron tunes, and linear $X-Y$ couplings, respectively. First- and higher-order coefficients of the series in Eq. (1) are defined as general chromaticity.

According to the matrix formalism, the one-turn map for betatron motion in a ring including $X-Y$ couplings is

$$
M_{4}(\delta)=R(\delta) \cdot M_{\text {lin }}(\delta) \cdot R^{-1}(\delta),
$$

where $M_{\text {lin }}$ is a $4 \times 4$ block diagonalized matrix describing uncoupled betatron motion [14] and $R$ is the coupling matrix. $M_{\text {lin }}$ and $R$ are defined as

$$
\begin{gathered}
M_{\operatorname{lin}}(\delta)=\left(\begin{array}{cc}
M_{x} & 0 \\
0 & M_{y}
\end{array}\right) \\
R(\delta)=\left(\begin{array}{cc}
r_{0} I_{2} & -S_{2} R_{2}^{T}(\delta) S_{2} \\
-R_{2}(\delta) & r_{0} I_{2}
\end{array}\right),
\end{gathered}
$$

where

$$
\begin{gathered}
M_{u}=\left(\begin{array}{cc}
\cos \mu_{u}(\delta)+\alpha_{u}(\delta) \sin \mu_{u}(\delta) & \beta_{u}(\delta) \sin \mu_{u}(\delta) \\
-\gamma_{u}(\delta) \sin \mu_{u}(\delta) & \cos \mu_{u}(\delta)-\alpha_{u}(\delta) \sin \mu_{u}(\delta)
\end{array}\right) \quad u=x, y \\
R_{2}(\delta)=\left(\begin{array}{cc}
r_{1}(\delta) & r_{2}(\delta) \\
r_{3}(\delta) & r_{4}(\delta)
\end{array}\right) \\
I_{2}=\left(\begin{array}{ll}
1 & 0 \\
0 & 1
\end{array}\right) \quad S_{2}=\left(\begin{array}{cc}
0 & 1 \\
-1 & 0
\end{array}\right) .
\end{gathered}
$$

Here, $\mu_{u}(\delta)=2 \pi \nu_{u}(\delta)$ is the momentum-dependent one-turn phase advance. Suppose that the chromaticity is related to a Hamiltonian

$$
H_{I}\left(x, p_{x}, y, p_{y}, \delta\right)=\sum_{n=1}^{\infty}\left(a_{n} x^{2}+b_{n} x p_{x}+c_{n} p_{x}^{2}+e_{n} x p_{y}+f_{n} p_{x} y+g_{n} p_{x} p_{y}+u_{n} y^{2}+v_{n} y p_{y}+w_{n} p_{y}^{2}\right) \delta^{n},
$$

where the coefficients $a_{n}$ to $w_{n}$ represent the chromatic effects and are related to the chromaticity coefficients in Eq. (1). The chromatic Hamiltonian in Eq. (8) results in a perturbative transformation of $M_{H}(\delta)$ for betatron motion. If the matrices obtained from the matrix formalism and by the Hamiltonian perturbative approach are equalized, a series of equations can be used to determine the coefficients in Eq. (8):

$$
M_{4}(\delta)=M_{4}(0) \cdot M_{H}(\delta) .
$$

After calculating the coefficients in Eq. (8) [11], we can use this Hamiltonian to construct a symplectic map for both betatron and synchrotron motions. Detailed formulas for this symplectic map are given in Sec. IIIC.

\section{MODEL FOR PARTICLE TRACKING}

\section{A. One-turn linear transformation}

To study the beam-beam effect with chromaticity, we select the observation location at interaction point (IP). The particle coordinates in six-dimensional (6D) phase space are defined as

$$
X=\left(x, p_{x}, y, p_{y}, z, \delta\right)^{T} .
$$


The one-turn linear transfer matrix at the IP is set as

$$
M_{0}=\left(\begin{array}{ccc}
M_{x} & 0 & 0 \\
0 & M_{y} & 0 \\
0 & 0 & M_{z}
\end{array}\right),
$$

where

$$
\begin{gathered}
M_{u}=\left(\begin{array}{cc}
\cos \mu_{u 0} & \beta_{u 0} \sin \mu_{u 0} \\
-\sin \mu_{u 0} / \beta_{u 0} & \cos \mu_{u 0}
\end{array}\right) \quad u=x, y \\
M_{z}=\left(\begin{array}{cc}
\cos \mu_{z 0} & -\beta_{z 0} \sin \mu_{z 0} \\
\sin \mu_{z 0} / \beta_{z 0} & \cos \mu_{z 0}
\end{array}\right)
\end{gathered}
$$

and

$$
\begin{gathered}
\mu_{u 0}=2 \pi \nu_{u 0} \quad u=x, y, z \\
\beta_{z 0}=\frac{c|\eta|}{\nu_{z} \omega_{0}},
\end{gathered}
$$

where $c$ is the speed of light, $\eta$ is the phase slip factor, and $\omega_{0}$ is the angular revolution frequency. Here, we assume that the alpha function and dispersion function at the IP are equal to zero. Linear $X-Y$ couplings are also set to zero, because in practical beam operation continuous linear optics corrections are usually carried out and these parameters mentioned above are tuned to be sufficiently small to achieve good matching. Meanwhile, the effect of linear couplings on luminosity is quite clear and should be removed from the simulations since we want to focus on the effect of the chromaticity on luminosity.

\section{B. Map of beam-beam interaction}

Both weak-strong and strong-strong beam-beam codes are available at KEK. The map of beam-beam interaction in these codes is also symplectic. The longitudinal variables, $z$ and $\delta$, are treated as dynamical variables; that is, they are not simple functions of $s$, such as $\sin \mu_{z} s / L$. The transverse beam-beam force is evaluated by solving a $2 \mathrm{D}$ Poisson equation or by using the Basseti-Erskine formula for a 2D Gaussian distribution, since beam particles are ultrarelativistic. The transverse beam-beam force depends on $z$ because of the beta variation near the IP. This implies that the beam-beam interaction contains a longitudinal force; otherwise, the symplectic condition in 6D phase space would not be guaranteed. For detailed discussions on the derivation of the maps, refer to Refs. [2,15,16].

It is noteworthy that in both weak-strong and strongstrong beam-beam codes, the crossing angle at the IP can be set to $11 \mathrm{~mm}$ rad or zero, corresponding to the cases of the crab cavities being off or on. Again, this makes it convenient to examine the effect of chromaticity in the cases of collisions when the crab cavities are on and off.

\section{Map of general chromaticity}

Since the beam-beam interaction is symplectic in 6D phase space, the chromaticity should be treated in the same manner; i.e., the longitudinal variables should be treated as dynamical variables. It is insufficient merely to use the $4 \times$ 4 matrix formalism in which the Twiss parameters are expanded for $\delta$. The chromaticity should be expressed by a symplectic map in $6 \mathrm{D}$ phase space. The chromatic Hamiltonian in Eq. (8) satisfies the need for constructing such a map.

For simplicity, series truncated to the third order of Eqs. (1) and (8) were used in constructing the map for chromaticity. This is reasonable because the contribution of higher-order chromaticity is usually small. Another reason for this is the fact that higher-order chromaticity could not be measured easily or calculated accurately through particle tracking as is demonstrated in Sec. IV. In practical simulations, the convergence properties of such a truncation were also verified. Using the Hamiltonian in Eq. (8) up to third order, the explicit transformation of chromatic perturbation for both betatron and synchrotron motions is expressed [11] as

$$
\begin{gathered}
\bar{p}_{x}=\frac{(1+V)\left(p_{x}-A x-D y\right)-E\left(p_{y}-U y-D x\right)}{(1+B)(1+V)-E F} \\
\bar{p}_{y}=\frac{p_{y}-U x-D y-F \bar{p}_{x}}{1+V} \\
\bar{x}=(1+B) x+C \bar{p}_{x}+F y+G \bar{p}_{y} \\
\bar{y}=(1+V) y+W \bar{p}_{y}+E y+G \bar{p}_{x} \\
\bar{\delta}=\delta
\end{gathered}
$$

$$
\begin{aligned}
\bar{z}= & z+A^{\prime}(\delta) x^{2}+B^{\prime}(\delta) x \bar{p}_{x}+C^{\prime}(\delta) \bar{p}_{x}^{2}+D^{\prime}(\delta) x y \\
& +E^{\prime}(\delta) x \bar{p}_{y}+F^{\prime}(\delta) \bar{p}_{x} y+G^{\prime}(\delta) \bar{p}_{x} \bar{p}_{y}+U^{\prime}(\delta) y^{2} \\
& +V^{\prime}(\delta) y \bar{p}_{y}+W^{\prime}(\delta) \bar{p}_{y}^{2}
\end{aligned}
$$

where

$$
\begin{array}{rlrl}
A(\delta) & =\sum_{i=1}^{3} a_{n} \delta^{n} & B(\delta) & =\sum_{i=1}^{3} b_{n} \delta^{n} \\
C(\delta) & =\sum_{i=1}^{3} c_{n} \delta^{n} & D(\delta) & =\sum_{i=1}^{3} d_{n} \delta^{n} \\
E(\delta) & =\sum_{i=1}^{3} e_{n} \delta^{n} & F(\delta) & =\sum_{i=1}^{3} f_{n} \delta^{n} \\
G(\delta) & =\sum_{i=1}^{3} g_{n} \delta^{n} & U(\delta) & =\sum_{i=1}^{3} u_{n} \delta^{n} \\
V(\delta) & =\sum_{i=1}^{3} v_{n} \delta^{n} & W(\delta) & =\sum_{i=1}^{3} w_{n} \delta^{n}
\end{array}
$$


and the variables with prime symbols in Eq. (21) denote differentiation with respect to $\delta$. It is noteworthy that the Hamiltonian expressed in Eq. (8) always exists for any given chromaticity as in Eq. (1). Further, the map represented by Eqs. (16)-(21) is always symplectic. This makes it convenient to verify different kinds of chromaticities and determine how adversely they will affect the beam emittance and luminosity. This is explained in greater detail in Sec. VI.

The map for chromaticity can be constructed by another direct method, in which expansion of the series of the Hamiltonian, as expressed in Eq. (8), is not required [11]. A direct map obtained by this method was also implemented in simulation codes. If the optics is not highly distorted by large chromaticities of alpha and beta functions, no major difference is found between these two methods, as was expected from the impact on beam dynamics.

\section{Map of radiation damping and quantum excitation}

Radiation damping and quantum excitation are added using transformation on the normal mode $X_{i}$ of the oneturn transfer matrix $M_{0}[16]$ as

$$
X_{i}=\left(\begin{array}{ccc}
D_{x} & 0 & 0 \\
0 & D_{y} & 0 \\
0 & 0 & D_{z}
\end{array}\right) X_{i}+\left(\begin{array}{ccc}
\chi_{x} & 0 & 0 \\
0 & \chi_{y} & 0 \\
0 & 0 & \chi_{z}
\end{array}\right) \hat{F}_{i},
$$

where

$$
D_{u}=\left(1-d_{u}\right) I_{2} \quad \chi_{u}=\sqrt{2 \epsilon_{u} d_{u}} I_{2} \quad u=x, y, z,
$$

$d_{u}=1 / \tau_{u}$ is the damping rate in one revolution, and $\tau_{u}$ is the damping time in the unit of turns, as shown in Table V. $\hat{F}_{i}$ is a vector of Gaussian random numbers with unit variance.

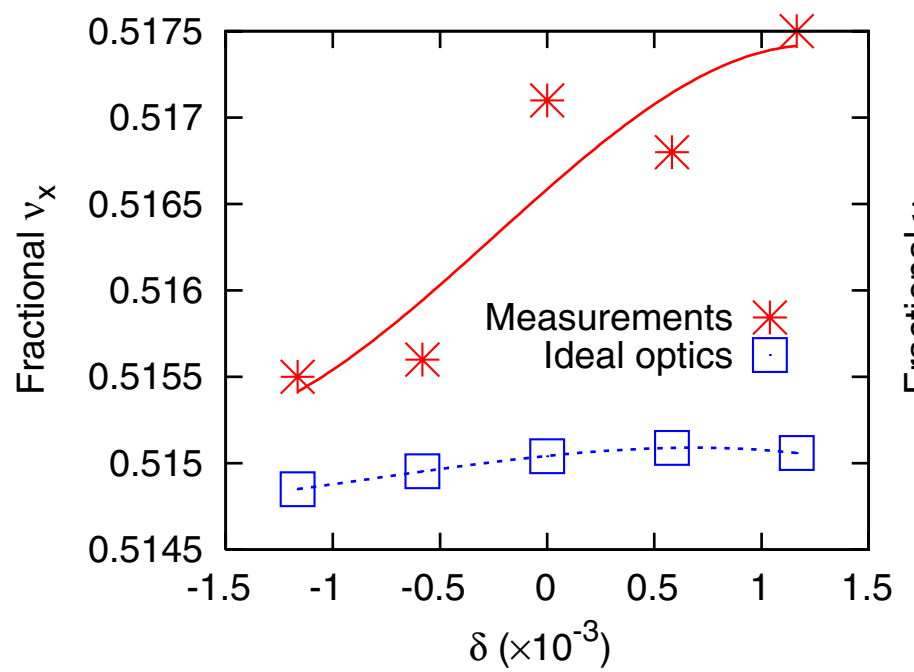

Finally, the one-turn map of the beam-beam interaction with chromaticity is constructed as

$$
M=M_{\mathrm{rad}} \circ M_{\mathrm{chr}} \circ M_{\mathrm{bb}} \circ M_{0}
$$

where $M_{\mathrm{bb}}, M_{\mathrm{chr}}$, and $M_{\mathrm{rad}}$ are maps for the beam-beam interaction, chromatic perturbation, and radiation damping and quantum excitation, respectively. Equation (25) also shows that in simulations, we can simply enable or disable the transformation for chromaticity and compare the luminosity performances. This is explained in greater detail in Sec. V. At the same time, we can also study synchrobetatron resonances in the presence of chromaticity by using the same code but disabling the beam-beam map.

\section{FIRST MEASUREMENT OF GENERAL CHROMATICITY AT KEKB}

At KEKB, intensive study on the effects of general chromaticity has been motivated by dedicated beam measurements. The experimental data, together with data from SAD modeling, were used in consequent simulations. The first systematic measurement of general chromaticity at the IP of KEKB HER was performed on October 27, 2008. We introduce the measurement results in this section.

Beta function with momentum deviation can be measured by changing the rf frequency of the accelerating cavities [17]. Then, the chromaticity of the beta function is calculated by fitting the data to a polynomial. The same method can be extended to measure the chromaticity of the alpha function, betatron tunes, dispersion, and $X-Y$ couplings. The measurement results are shown in Figs. 1-3. The momentum compaction of the KEKB HER was $3.38 \times 10^{-4}$, and the nominal $\mathrm{rf}$ frequency was $508.886 \mathrm{MHz}$. In each figure, the measurement results are also compared with results of the SAD calculation with ideal optics. The corresponding chromaticity was

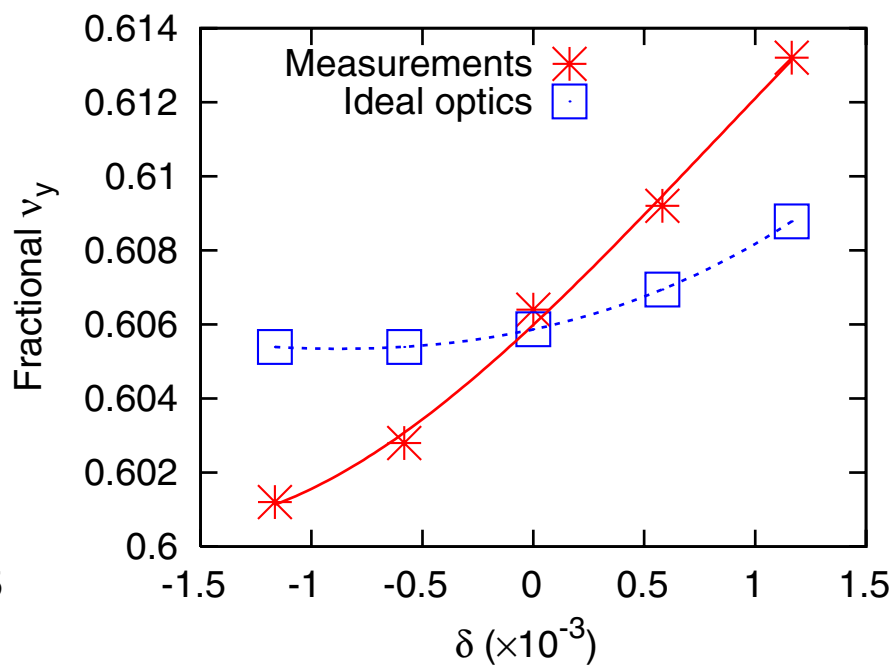

FIG. 1. (Color) Betatron tunes as a function of momentum deviation. The rf frequency shifts from -200 to $200 \mathrm{~Hz}$ with a $100 \mathrm{~Hz}$ interval. The solid and dashed lines represent third-order polynomial fittings. 

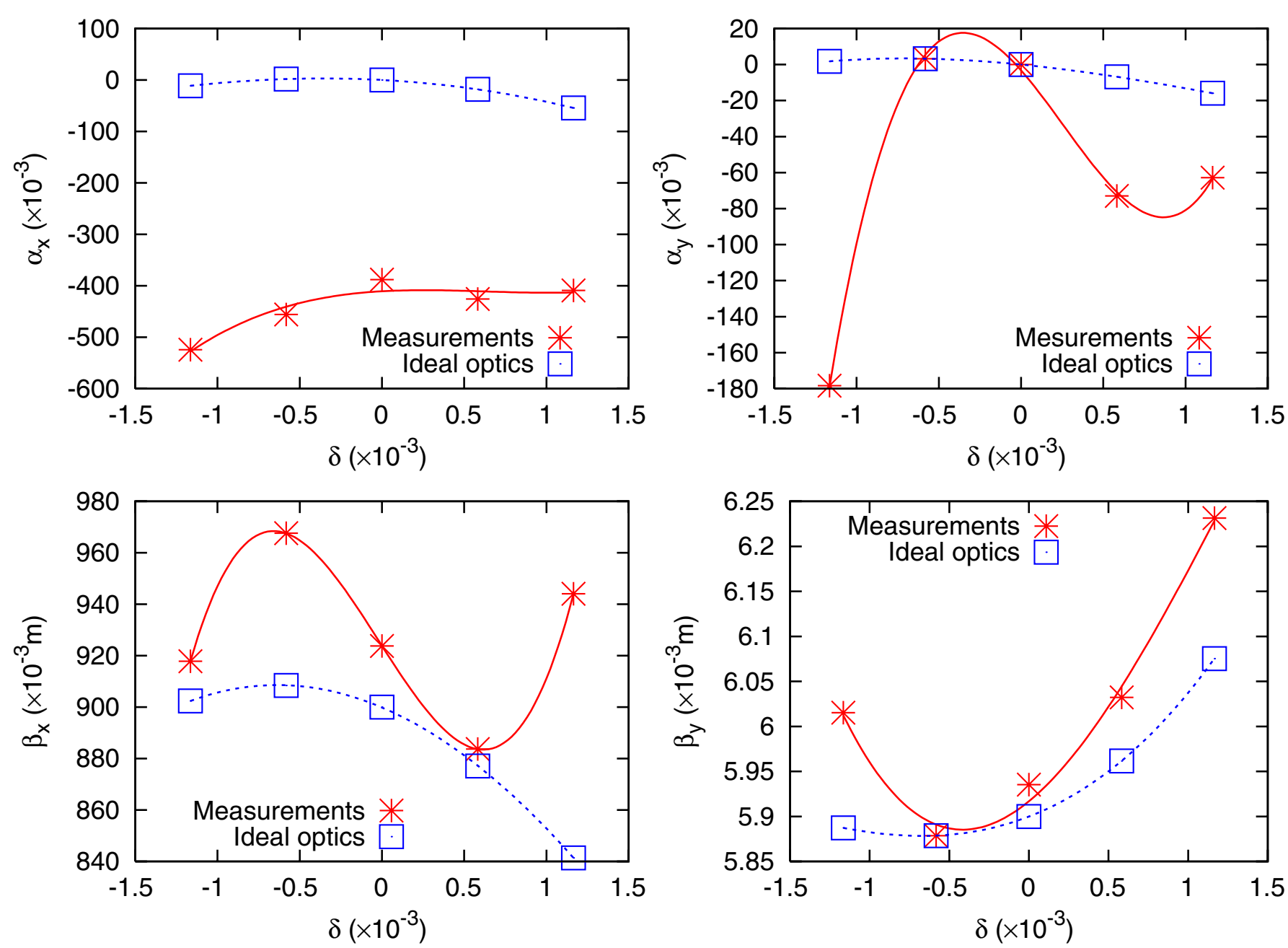

FIG. 2. (Color) Alpha and beta functions at the IP as a function of momentum deviation. The rf frequency shifts from -200 to $200 \mathrm{~Hz}$ with a $100 \mathrm{~Hz}$ interval. The solid and dashed lines represent third-order polynomial fittings.

calculated through polynomial fittings; the results are listed in Tables I and II. The coefficients of the chromatic Hamiltonian were also calculated, as shown in Table III.

Figure 1 shows the tune shift versus relative momentum deviation. The calculated tune chromaticity indicates a significant discrepancy between the ideal optics and the measurement. In practice, the normal tune chromaticity may be changed by tuning optimization to improve the beam lifetime and luminosity during machine operation. Another fact is that machine errors can also contribute to additional tune chromaticity.

The measured chromaticity of the alpha and beta functions is also quite different from that of ideal optics, as shown in Tables I and II and Fig. 2. Specifically, the measured second- and third-order chromaticities are much larger than those of ideal optics. We found that such large chromaticities will cause strong optics distortion for off-momentum particles and, consequently, lead to unstable beams in beam-beam simulations. It implies that the measurement results were not convincingly reasonable. This may be due to the difficulties in measurements. The first difficulty was that the beta functions were hard to be measured when the betatron tunes were close to half integer. Second, the number of data for polynomial fittings was not enough due to limited beam time for machine study. Third, Twiss parameters with large momentum deviation, which corresponds to large rf frequency shift, could not be measured due to poor beam lifetime.

The most interesting fact is that the $X-Y$ couplings at the IP also vary with momentum deviation, even in the case of ideal optics, as shown in Fig. 3. More accurate measurements of chromatic $X-Y$ couplings were performed by Ohnishi et al. [18]. As discussed in Ref. [18], the chromatic $X-Y$ couplings without machine errors, considered as "natural chromatic coupling," may originate from the special magnets and the final focus quadrupole magnet in the IR region. In addition, both machine errors and continuous optics corrections for the purpose of luminosity optimization can lead to nonzero chromatic $X-Y$ couplings.

Actually, we used random error seedings to model the machine errors using SAD. We found that the chromaticity depends greatly on error seedings. Thus, statistics on dif- 

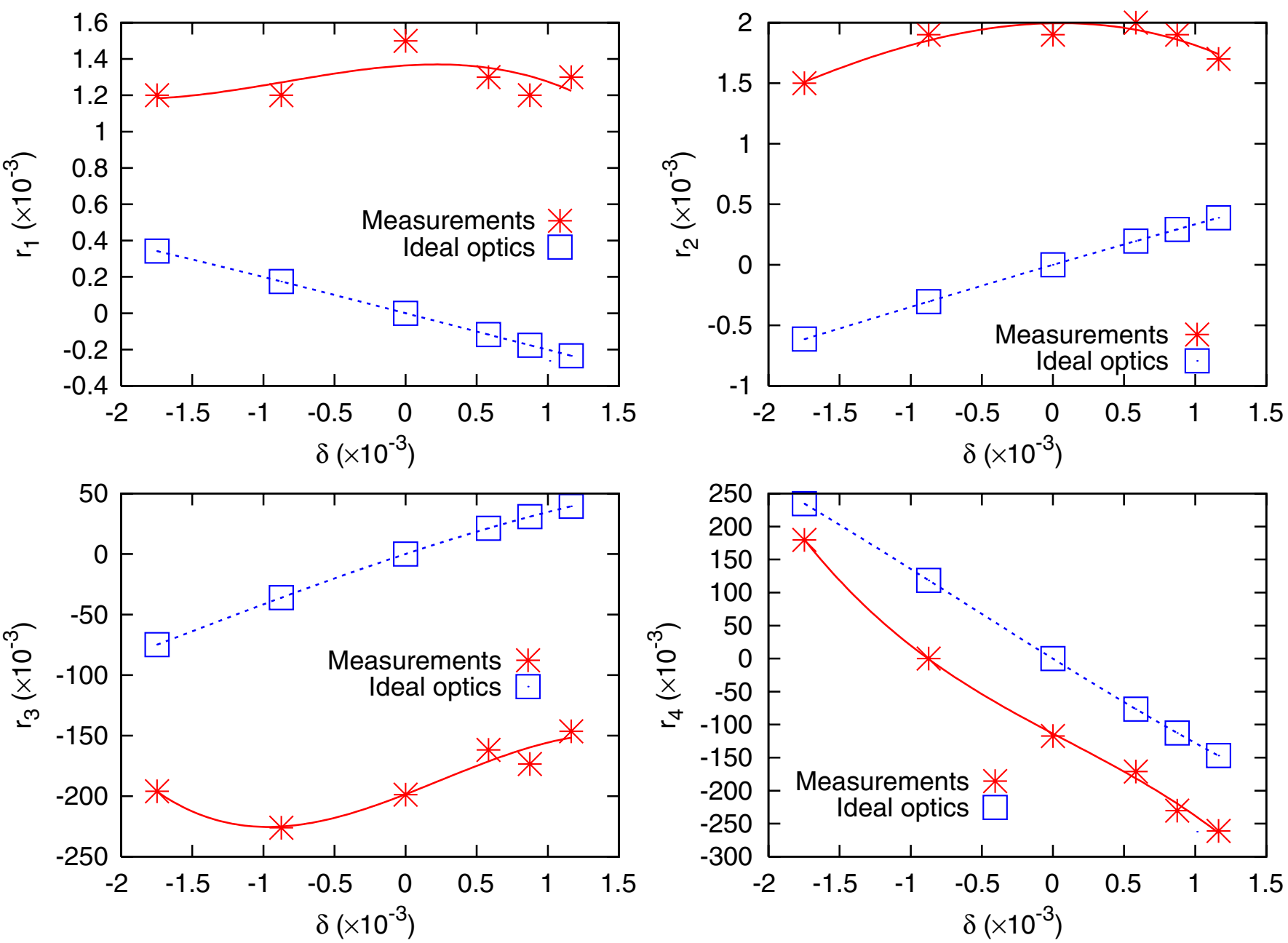

FIG. 3. (Color) $X-Y$ coupling parameters at the IP as a function of momentum deviation. The rf frequency shifts from -300 to $200 \mathrm{~Hz}$. The solid and dashed lines represent third-order polynomial fittings.

ferent seeds may be more reasonable estimations of the chromaticity. In practice, 1000 seeds were applied to an ideal optics at the KEKB HER. For each seed, we calculated all optics parameters. As examples, the distributions of $r_{31}$ and $r_{41}$ are shown in Fig. 4. The average values and

TABLE I. Chromaticity of ideal optics at KEKB HER.

\begin{tabular}{lccc}
\hline \hline Parameter & $i=1$ & $i=2$ & $i=3$ \\
\hline$\nu_{x i}$ & 0.126 & -64.6 & $-2.7 \times 10^{4}$ \\
$\nu_{y i}$ & 1.29 & 898 & $1.22 \times 10^{5}$ \\
$\alpha_{x i}$ & -16.9 & $-2.43 \times 10^{4}$ & $-1.22 \times 10^{6}$ \\
$\beta_{x i}$ & -27.1 & $-2.06 \times 10^{4}$ & $6.76 \times 10^{5}$ \\
$\alpha_{y i}$ & -9.01 & -5207 & $1.03 \times 10^{6}$ \\
$\beta_{y i}$ & 0.0682 & 60 & 9240 \\
$r_{1 i}$ & -0.2 & -0.894 & 1578 \\
$r_{2 i}$ & 0.342 & -6.41 & -601 \\
$r_{3 i}$ & 38.8 & -3469 & $-6.34 \times 10^{5}$ \\
$r_{4 i}$ & -134 & 4156 & $2.3 \times 10^{6}$ \\
\hline \hline
\end{tabular}

their variations for $r_{1 i}$ to $r_{4 i}$ at the IP are summarized in Table IV.

In summary, the measured chromaticity usually disagrees with the chromaticity in the optics models, as shown

TABLE II. Chromaticity obtained from first beam measurement at KEKB HER.

\begin{tabular}{lccr}
\hline \hline Parameter & $i=1$ & $i=2$ & \multicolumn{1}{c}{$i=3$} \\
\hline$\nu_{x i}$ & 1.09 & -126 & $-1.69 \times 10^{5}$ \\
$\nu_{y i}$ & 5.61 & 842 & $-3.37 \times 10^{5}$ \\
$\alpha_{x i}$ & 18.0 & $-4.4 \times 10^{4}$ & $2.31 \times 10^{7}$ \\
$\beta_{x i}$ & -99.8 & 5240 & $8.19 \times 10^{7}$ \\
$\alpha_{y i}$ & -104 & $-8.7 \times 10^{4}$ & $1.13 \times 10^{8}$ \\
$\beta_{y i}$ & 0.145 & 150 & $-3.83 \times 10^{4}$ \\
$r_{1 i}$ & 0.0495 & -99.5 & $-3.94 \times 10^{4}$ \\
$r_{2 i}$ & 0.019 & -182 & $-1.95 \times 10^{4}$ \\
$r_{3 i}$ & 45.7 & 7860 & $-1.09 \times 10^{7}$ \\
$r_{4 i}$ & -114 & 4440 & $-1.52 \times 10^{7}$ \\
\hline \hline
\end{tabular}


TABLE III. First-order coefficients of chromatic Hamiltonian calculated from chromaticity at KEKB HER.

\begin{tabular}{lcc}
\hline \hline Parameter & Ideal optics & Measurement \\
\hline$a_{1}$ & 2.10 & 10.2 \\
$b_{1}$ & -1.31 & 0.47 \\
$c_{1}$ & -0.984 & -1.99 \\
$d_{1}$ & -22.1 & 33.5 \\
$e_{1}$ & 0.176 & 0.178 \\
$f_{1}$ & 65.5 & 56.2 \\
$g_{1}$ & -0.386 & -0.533 \\
$u_{1}$ & 502 & 5359 \\
$v_{1}$ & -8.78 & -59.6 \\
$w_{1}$ & 0.0303 & 0.0229 \\
\hline \hline
\end{tabular}

in Tables I, II, and III. In KEKB rings, the chromaticity of tunes, alpha and beta functions at the IP was controllable and knobbed according to the luminosity performance. However, the chromaticity of the $X-Y$ couplings at the IP had not been controlled until the recent installation of skew sextupoles [18].

\section{BEAM-BEAM SIMULATIONS WITH CHROMATICITY}

From this section, we apply the data from measurement and SAD modeling to beam-beam simulations and study the effects of general chromaticity as a whole at KEKB. The main parameters used for beam-beam simulations at
KEKB are summarized in Table V. The tunes are similar to the operation tunes during the autumn run in 2008. To avoid the occurrence of the flip-flop effect, all parameters except for beam energy and bunch population, are selected to be equal for both rings.

Here we mainly present the simulation results using the first three orders of chromaticity of the ideal optics. We checked the second- and third-order chromaticities and found that, for the ideal optics, the contribution of these high-order chromaticities to luminosity is negligible. However, for the measurement data, the strong distortion due to large second- and third-order chromaticities of vertical alpha and beta functions (see Fig. 2) leads to unstable beams in tracking. Thus, high-order chromaticity obtained from measured data cannot be used in simulations. When the first-order chromaticity is taken into account, the ideal optics and measurement yield very similar luminosity degradation in weak-strong simulations (see Fig. 13). But a remarkable difference was observed in strong-strong simulations, as shown in Fig. 5. This may be explained by the presence of dynamic effects due to beam-beam interaction; that is, large chromaticity of the alpha and beta functions may worsen the dynamic effects and thus cause a more severe loss of luminosity at higher bunch currents.

For the weak-strong and strong-strong simulations, the numbers of macroparticles for one bunch with an initial Gaussian distribution were set to 1000 and 200000 , respectively. The macroparticles were tracked in 20000 turns

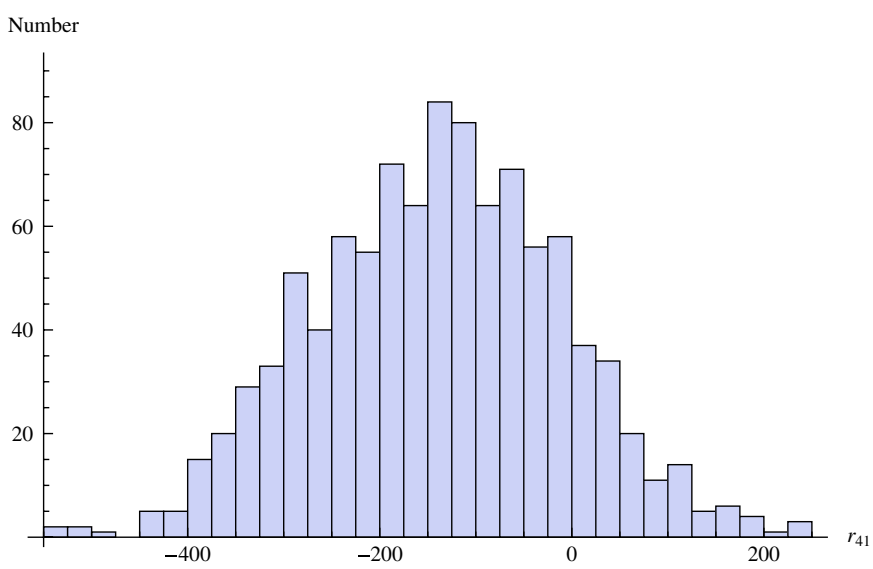

FIG. 4. (Color) Distributions of $r_{31}$ and $r_{41}$ with 1000 error seeds for KEKB HER.

TABLE IV. Average and variance of chromatic coupling parameters calculated from 1000 seeds of errors at KEKB HER.

\begin{tabular}{lccc}
\hline \hline Parameter & $i=1$ & $i=2$ & $i=3$ \\
\hline$r_{1 i}$ & $-0.21 \pm 0.25$ & $-2.2 \pm 21$ & $1600 \pm 7300$ \\
$r_{2 i}$ & $0.34 \pm 0.27$ & $-3.9 \pm 29$ & $-39 \pm 6000$ \\
$r_{3 i}$ & $37 \pm 140$ & $-3400 \pm 6000$ & $(-0.64 \pm 2.1) \times 10^{6}$ \\
$r_{4 i}$ & $-140 \pm 130$ & $5100 \pm 11000$ & $(2.6 \pm 2.1) \times 10^{6}$ \\
\hline \hline
\end{tabular}


TABLE V. Main parameters used for beam-beam simulations at KEKB. Apart from beam energy and bunch population, all parameters are set to be equal for the HER and LER.

\begin{tabular}{lccc}
\hline \hline Parameter & Description & $e^{+}$ & $e^{-}$ \\
\hline$E(\mathrm{GeV})$ & Beam energy & 3.5 & 8.0 \\
$C(\mathrm{~m})$ & Circumference & 3016.25 & 3016.25 \\
$N\left(10^{10}\right)$ & Bunch population & 10.0 & 4.375 \\
$\beta_{x}^{*}(\mathrm{~m})$ & Horizontal beta function at the IP & 0.9 & 0.9 \\
$\beta_{y}^{*}(\mathrm{~mm})$ & Vertical beta function at the IP & 6.0 & 6.0 \\
$\epsilon_{x}(\mathrm{~nm} \mathrm{rad})$ & Horizontal emittance & 18.0 & 18.0 \\
$\epsilon_{y}(\mathrm{~nm} \mathrm{rad})$ & Vertical emittance & 0.18 & 0.18 \\
$\nu_{x}$ & Horizontal tune & 44.5151 & 44.5151 \\
$\nu_{y}$ & Vertical tune & 41.6059 & 41.6059 \\
$\nu_{z}(\mathrm{~mm})$ & Synchrotron tune & 0.024 & 0.024 \\
$\sigma_{z}\left(10^{-4}\right)$ & Bunch length $\left(I_{b}=0\right)$ & 6.93 & 6.93 \\
$\sigma_{\delta}($ turns $)$ & Energy spread $\left(I_{b}=0\right)$ & 6.93 & 6.93 \\
$\tau_{x, y}($ turns $)$ & $x, y$ damping time & 4000 & 4000 \\
$\tau_{z}$ & $z$ damping time & 2000 & 2000 \\
\hline \hline
\end{tabular}

to obtain equilibrium distributions. Beam envelope parameters of the colliding bunches were numerically calculated and then used to calculate luminosity [19]. Specific luminosity as a function of bunch current product is shown in Fig. 5. Weak-strong and strong-strong simulations were performed for colliding beams with crab cavities turned on and off. In the case of crab-off collision, the chromaticity shows a relatively small effect on luminosity. However, in the case of the crab cavities turned on, the figure clearly shows that luminosity degradation occurs because of chromaticity. The weak-strong simulations show that an average of 5\% degradation occurs, which does not depend on bunch current up to $1.6 \mathrm{~mA}$. On the other hand, the strongstrong simulation shows that the degradation is larger and

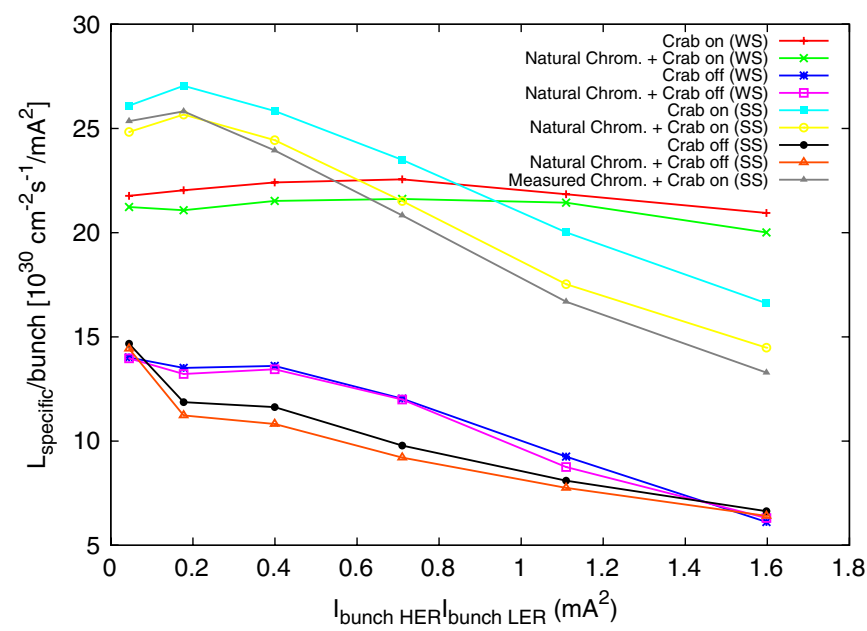

FIG. 5. (Color) Specific luminosity as a function of current product for KEKB. The natural chrom. and measured chrom. indicate natural chromaticity and measured chromaticity calculated from ideal optics and measurement data at KEKB HER, respectively. The WS and SS represent weak-strong and strongstrong simulations, respectively. slightly dependent on bunch current. In particular, a degradation of around $10 \%$ occurs for a current product of $1.1 \mathrm{~mA}^{2}$.

It is also noteworthy that in the case of crab cavities turned on, the slope of specific luminosity in strong-strong simulations is much steeper than that in weak-strong simulations. Specifically, there is a large discrepancy in the luminosity at a low bunch current product of around $0.2 \mathrm{~mA}^{2}$. This is also a consequence of the dynamic beta effect [20]. In the case of ultralow bunch currents as well, both the weak-strong and the strong-strong simulations yield the same luminosity.

\section{SCAN WITH FIRST-ORDER CHROMATICITY}

To identify the effect of different terms of chromaticity on luminosity and beam size, we fixed the tunes to $\nu_{x}=$ 44.5151 and $\nu_{y}=41.6059$ and scanned each term on a large scale in weak-strong simulations. Only the first-order chromaticities of Twiss parameters and $X-Y$ couplings were scanned since they are usually dominant.

The statistical errors of calculated luminosity were reduced by averaging the values of luminosity and beam sizes over the last 8000 turns. From Fig. 6, we can observe that the luminosity degradation was quite small when we applied the first-order chromaticities of alpha and beta functions at the IP in simulations. Obvious luminosity degradation was observed when the chromaticity was large; however, this seems impossible in reality. This observation does not quite agree with the single knobs of beam tuning at KEKB, as shown in Fig. 7. In practice, such single knobs were designed to improve the beam lifetime. However, it was found that luminosity is also sensitive to the chromaticity of alpha and beta functions at IP. One hypothesis is that the application of a single knob has side effects correlated to synchrobeta resonances [21]. It should be noted that these parameters have been scanned on a 

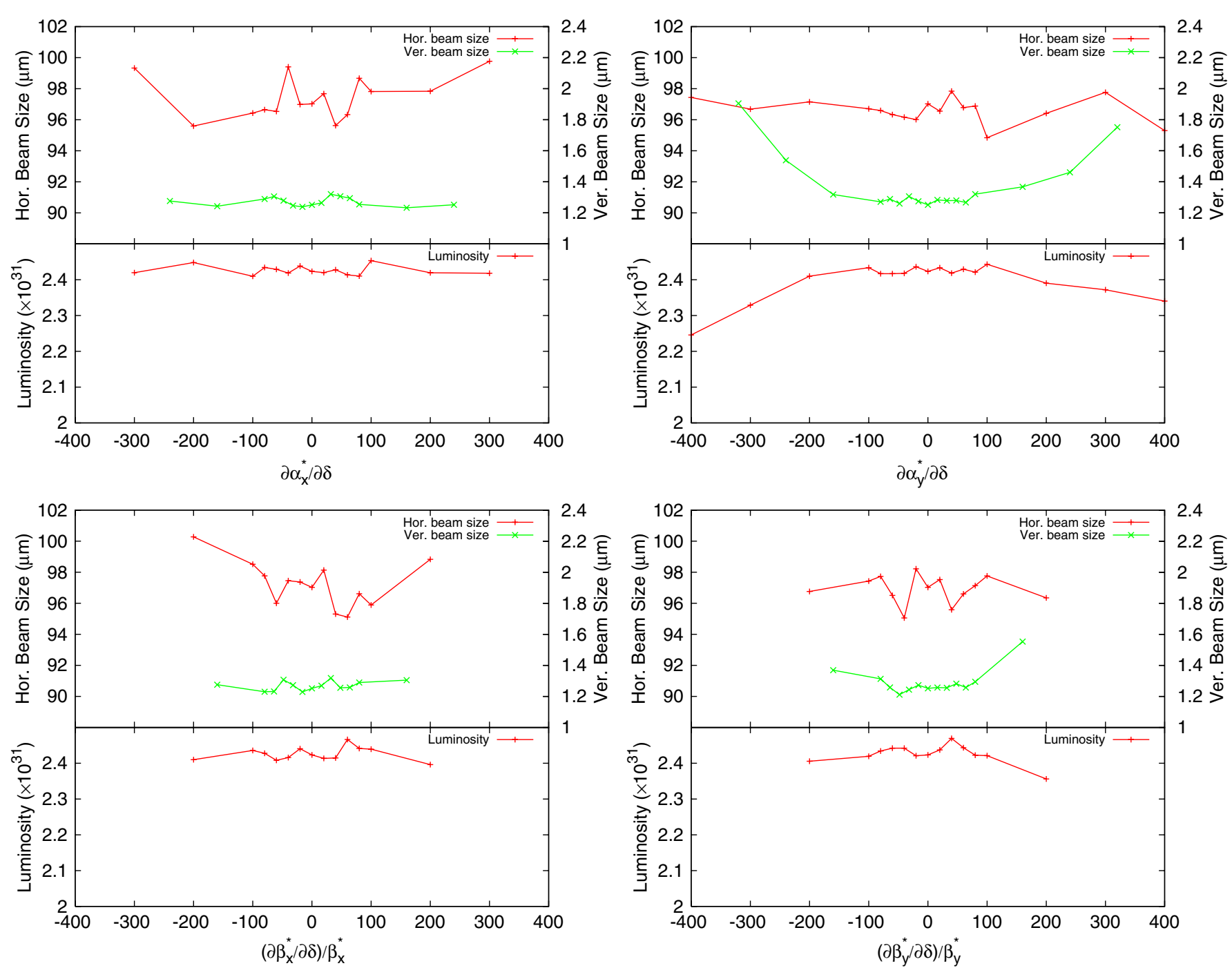

FIG. 6. (Color) Scan of first-order chromaticities of alpha and beta functions at the IP with beam-beam interaction. The beam suddenly becomes unstable when $\left|\partial \alpha_{x}^{*} / \partial \delta\right|>300,\left|\partial \alpha_{y}^{*} / \partial \delta\right|>400,\left|\left(\partial \beta_{x}^{*} / \partial \delta\right) / \beta_{x}^{*}\right|>200$, and $\left|\left(\partial \beta_{y}^{*} / \partial \delta\right) / \beta_{y}^{*}\right|>200$.

much larger scale in simulations than in tuning knobs. Further, in simulations, when these parameters have very large values, the beam suddenly becomes unstable due to severe optics distortion for off-momentum particles (see Fig. 6).

Figure 8 shows the scan results of chromatic $X-Y$ couplings at the IP. Following the definitions in Ref. [21], we adopted the following normalization for convenience of comparison:

$$
\left(\begin{array}{ll}
r_{1 N}^{*} & r_{2 N}^{*} \\
r_{3 N}^{*} & r_{4 N}^{*}
\end{array}\right)=\left(\begin{array}{cc}
r_{1}^{*} \sqrt{\beta_{x}^{*} / \beta_{y}^{*}} & r_{2}^{*} / \sqrt{\beta_{x}^{*} \beta_{y}^{*}} \\
r_{3}^{*} \sqrt{\beta_{x}^{*} \beta_{y}^{*}} & r_{4}^{*} \sqrt{\beta_{y}^{*} / \beta_{x}^{*}}
\end{array}\right) .
$$

As shown in Fig. 8, a comparison between scans of the chromaticities of alpha and beta functions reveals that there is a very clear correlation between luminosity degradation and chromatic $X-Y$ couplings. For example, with some simple calculations using the chromaticity given in Table I, a value of $r_{41}=-134$, corresponding to $\partial r_{4 N}^{*} / \partial \delta=-11$, causes a luminosity degradation of around $4 \%$. We remember that the weak-strong simulation predicts $5 \%$ in total. This let us conclude that the luminosity degradation shown in Fig. 5 is mainly associated with $r_{41}=\partial r_{4}^{*} / \partial \delta$. But we emphasize that it is not a general conclusion. In fact, the contributions of chromatic $X-Y$ couplings to the perturbative Hamiltonian in Eq. (8) are weighted by betatron tunes.

The momentum dependence of second-order betatron tunes may be a concern because it may affect luminosity. Therefore, we also scanned the second-order chromaticity of horizontal tune. The vertical tune was fixed at 41.6059, and the horizontal tune was varied from 44.505 to 44.52 . The results of scanning $\nu_{x 2}$ are shown in Fig. 9. Negative $\nu_{x 2}$ drives particles with high momentum deviation close to half-integer resonance, and positive $\nu_{x 2}$ drives particles 

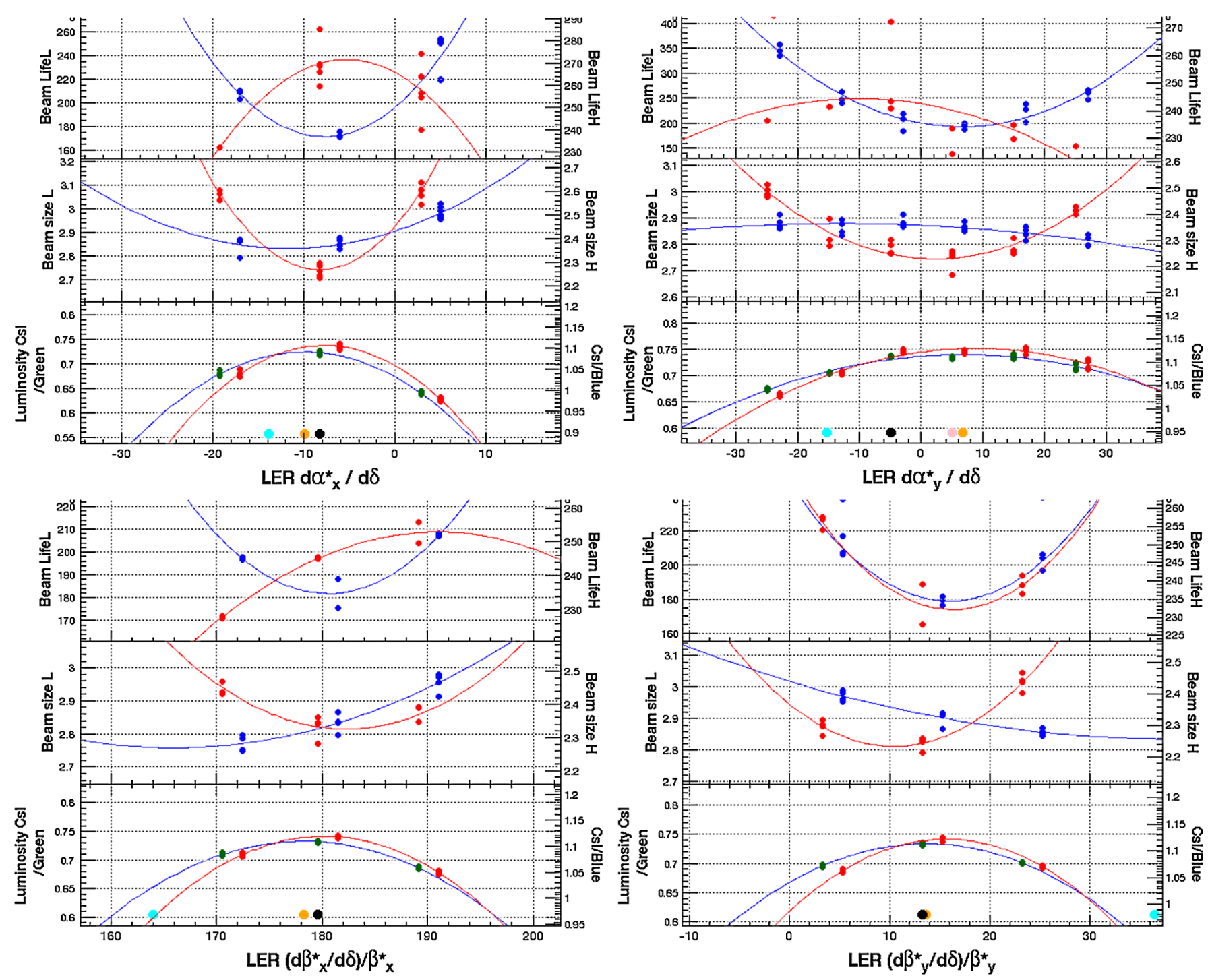

FIG. 7. (Color) Single knobs of chromaticities of alpha and beta functions at the IP of KEKB LER. In each subfigure, the red and blue dots represent data acquired from the LER and HER, respectively. The solid red and blue lines represent polynomial fittings.

away from half-integer resonance. This characteristic explains why we observe asymmetric effects of chromaticity on beam size and luminosity in the figure. We also notice that when the horizontal tune is moving close to halfinteger resonance, the luminosity degradation worsens with nonzero $\nu_{x 2}$. The scan of second-order chromaticity of the vertical tune does not show notable luminosity degradation, because the vertical tune is much farther from half integer than the horizontal tune.

\section{TUNE DEPENDENCE OF CHROMATIC EFFECT}

In Secs. V and VI, we fixed the nominal tunes in all simulations. Apparently, the effect of chromaticity on luminosity is tune dependent; therefore, in this section, we present the results of tune scan on beam size and luminosity by using weak-strong simulations. Again, we consider the chromaticity of ideal optics, including first- to thirdorder terms. The fractional tune region was set from 0.505 to 0.995 for both $\nu_{x}$ and $\nu_{y}$. The tune step for the scan was set to 0.005 . For simplification of code development, we fixed the coefficients of the chromatic Hamiltonian to those calculated from the ideal optics with tune (44.5151, 41.6059). As follows from fixing the chromatic Hamiltonian, we do not guarantee that the chromaticity will be frozen when we change tunes in simulations.

A comparison between the tune scans with and without the map of chromaticity, shown in Figs. 10-12, leads to the following conclusions: (1) The area of high-luminosity regions with tunes near half-integer shrinks due to chromaticity (see Fig. 10). (2) Luminosity degradation is primarily associated with a blowup of the vertical beam size and the degradation rate depends on tunes (see Figs. 11 and 12). (3) The width of the stop band at resonance 

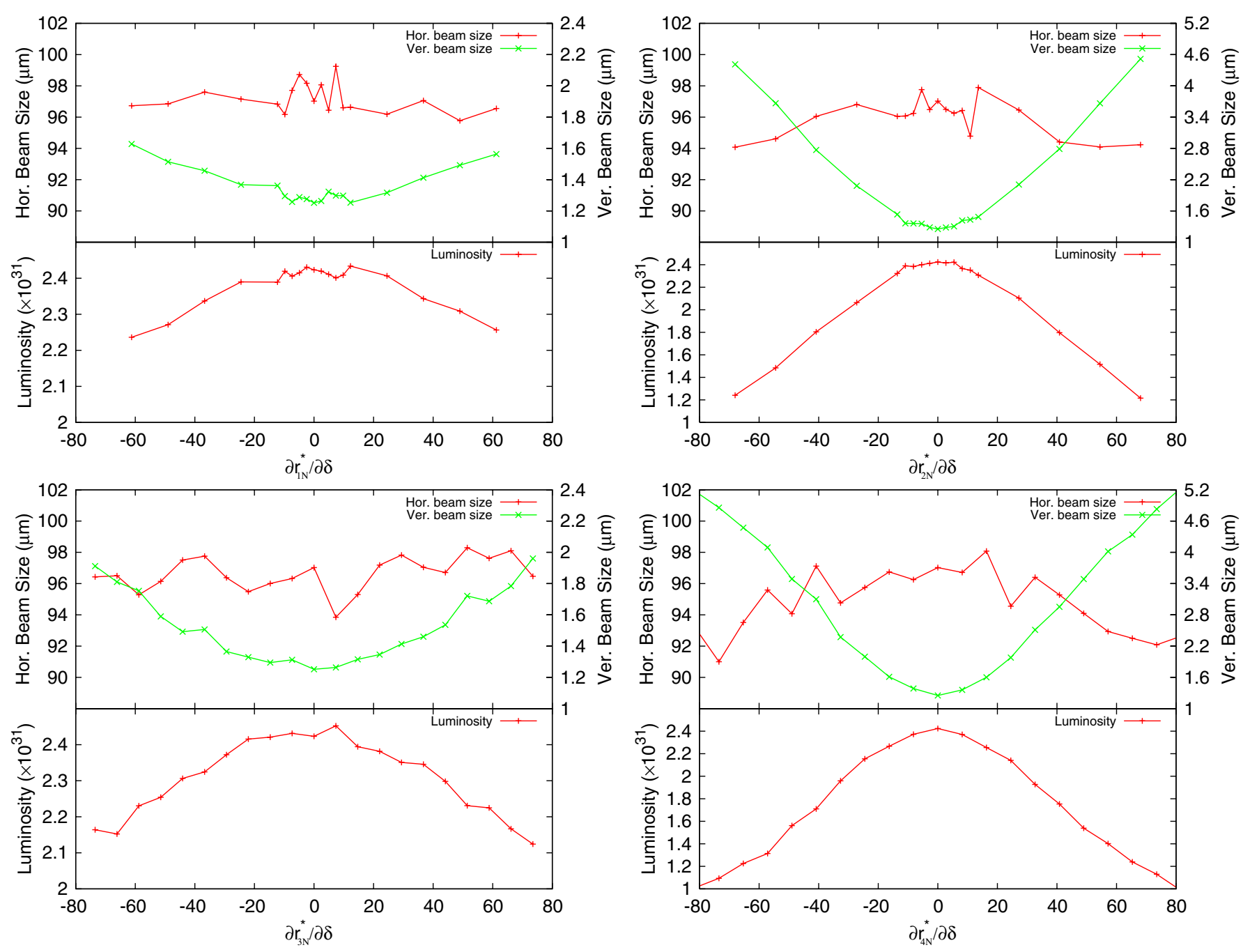

FIG. 8. (Color) Scan of first-order chromaticity of $X-Y$ couplings at the IP.

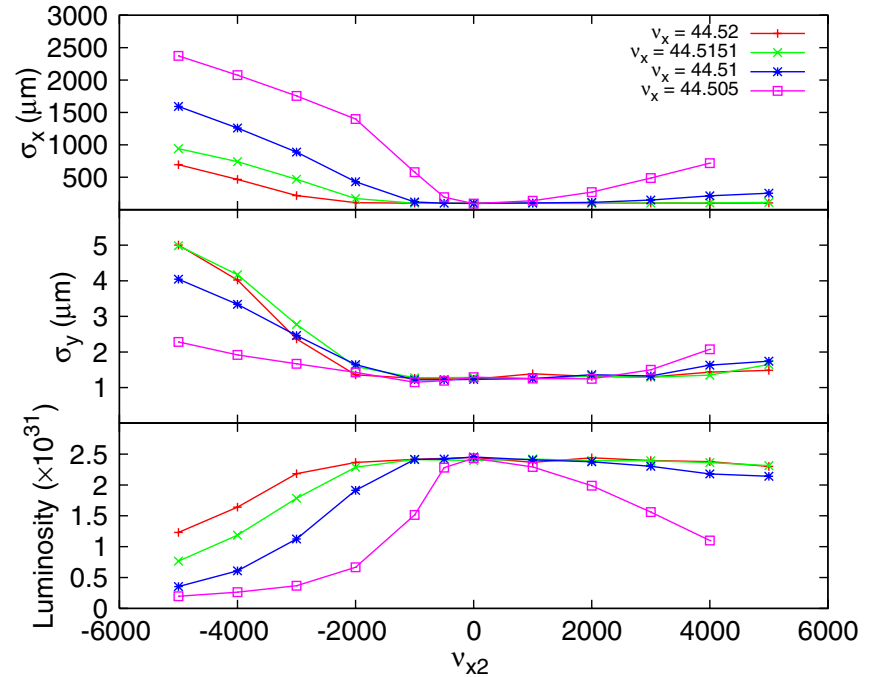

FIG. 9. (Color) Scan of second-order chromaticity of horizontal tune.
$\nu_{x}-\nu_{y}+\nu_{z}=N$, where $N$ is an integer, is dramatically enhanced by chromaticity and leads to the blowup of the vertical beam size (see Figs. 10 and 12).

As a special case of $\nu_{y}=41.60$, the tune scan with $\nu_{x}$ from 44.51 to 44.71 was performed in weak-strong beambeam simulations with and without chromaticity (Fig. 13). For convenience of comparison, we only used first-order chromaticity in each simulation. Comparing with the results of only beam-beam effect, the luminosity degradation and beam size blowup due to chromaticity obviously change with tunes. The beam-beam effect dominates the blowup of the horizontal beam size; and the chromaticity can remarkably dilute vertical emittance and induce luminosity degradation. From Fig. 13, we also observed that luminosity performances in the cases of first-order chromaticities of the ideal optics and measured optics, as shown in Tables I and II, are very similar. This is because $r_{41}$ is dominant among the first-order chromaticities, and the 

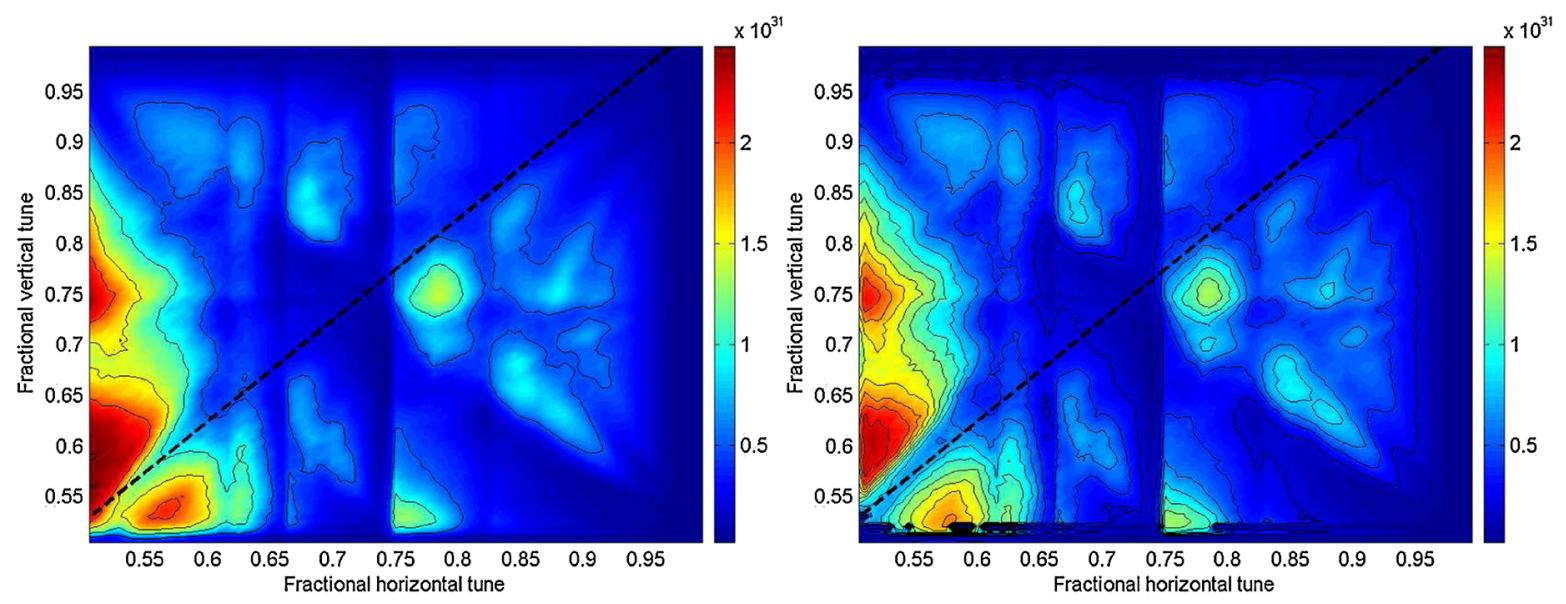

FIG. 10. (Color) Luminosity scans in tune space with and without chromaticity. The dashed line in each figure represents the synchrobeta resonance at $\nu_{x}-\nu_{y}+\nu_{z}=N$ (left: only beam-beam interaction; right: beam-beam interaction plus chromaticity of ideal optics).
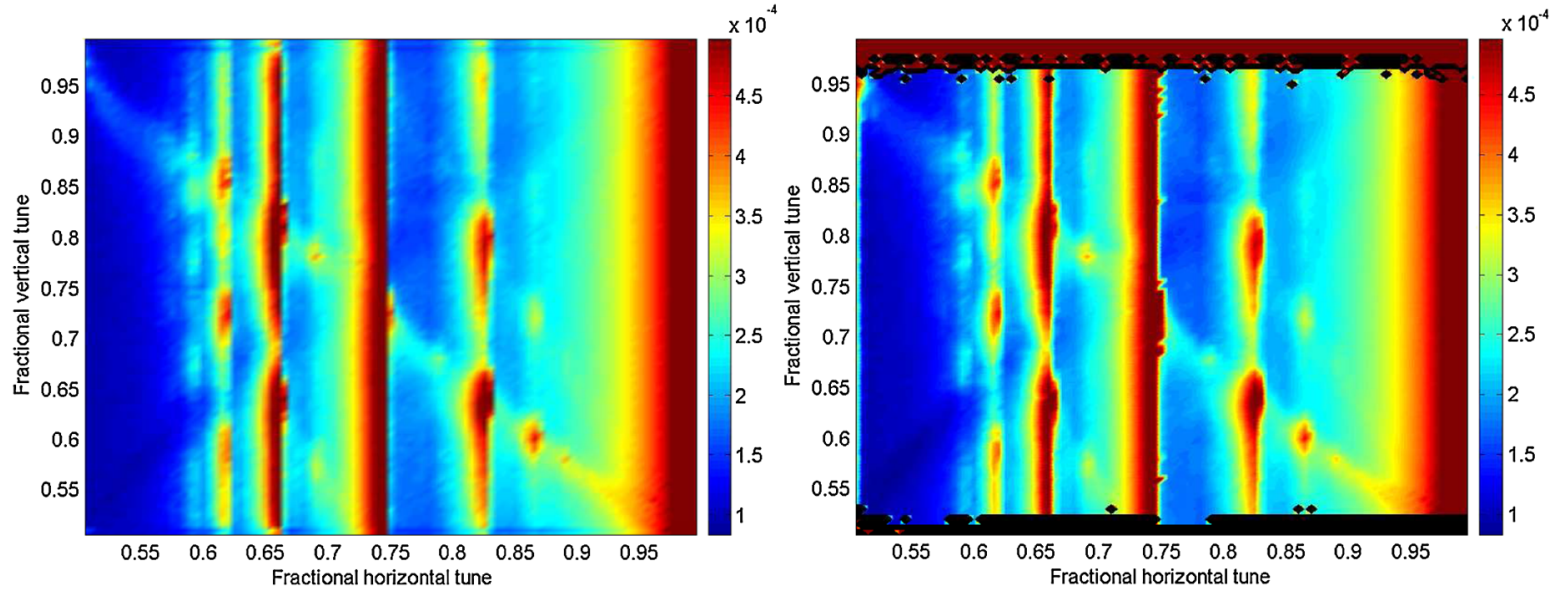

FIG. 11. (Color) Horizontal beam size scans in tune space (left: only beam-beam interaction; right: beam-beam interaction plus chromaticity of ideal optics; the black areas indicate that beam is unstable because of strong synchrobeta resonances).
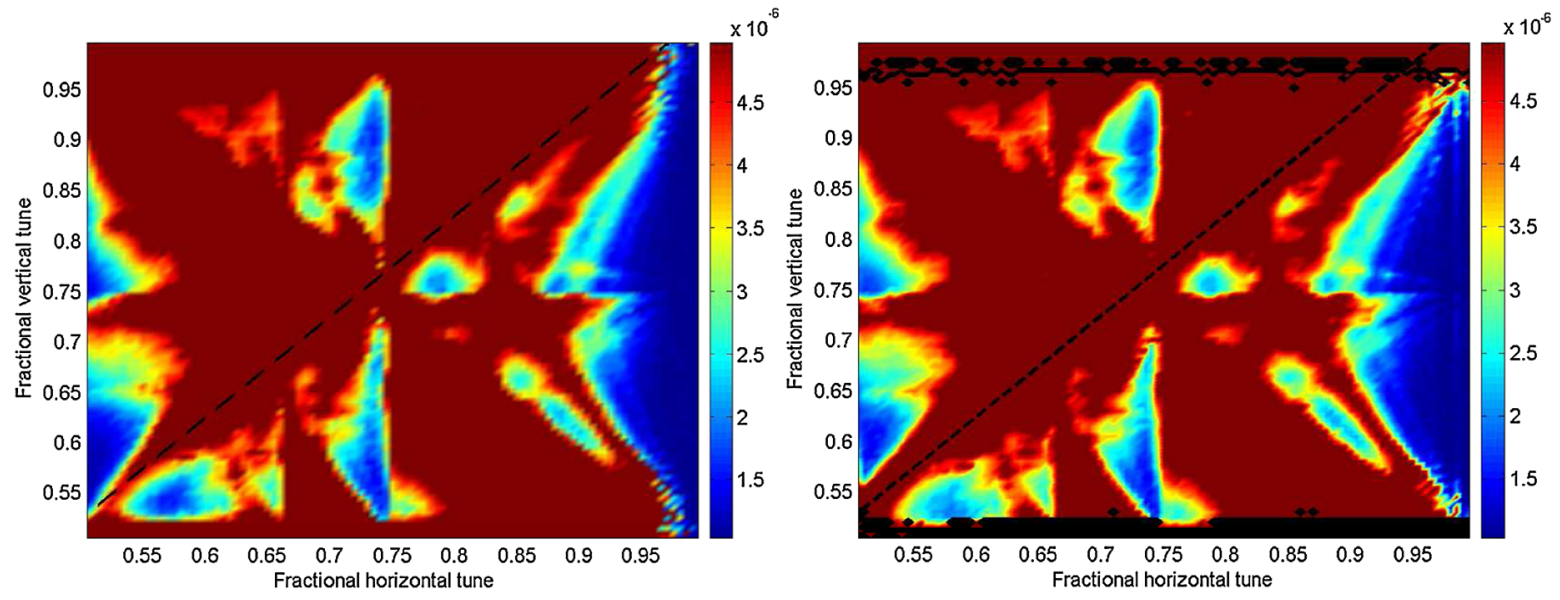

FIG. 12. (Color) Vertical beam size scans in tune space. The colors are set to dark red in areas where the beam sizes are larger than $5 \mu \mathrm{m}$. The dashed line in each figure represents the synchrobeta resonance at $\nu_{x}-\nu_{y}+\nu_{z}=N$ (left: only beam-beam interaction; right: beam-beam interaction plus chromaticity of ideal optics; the black areas indicate that beam is unstable because of strong synchrobeta resonances). 


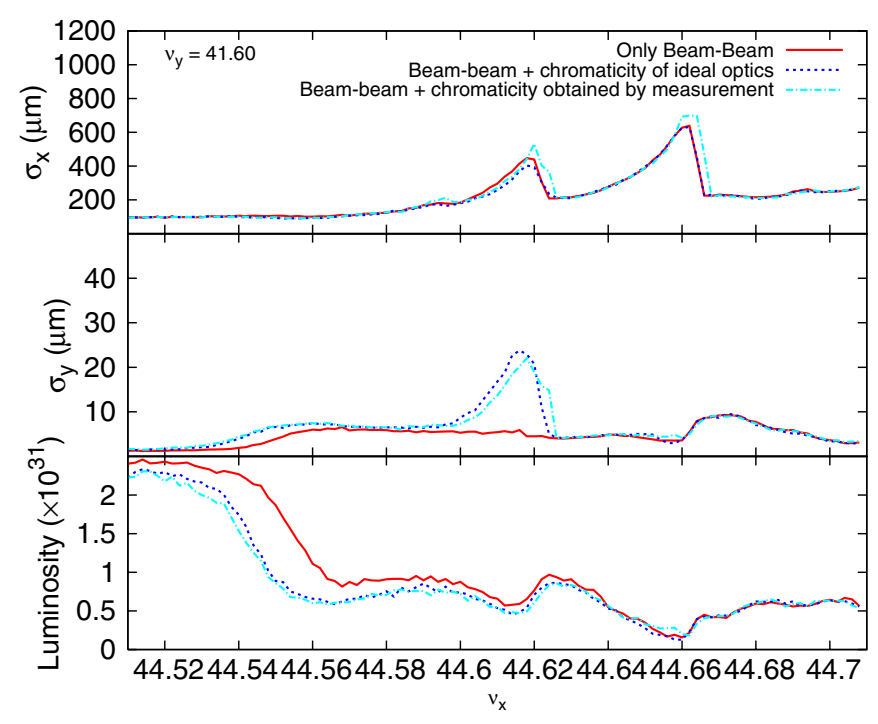

FIG. 13. (Color) Horizontal tune scan with beam-beam interaction only or plus first-order chromaticity. The first-order chromaticity was employed in the simulations.

values of $r_{41}$ of the ideal optics and measured optics are close to each other.

\section{SUMMARY AND DISCUSSIONS}

On the base of the chromaticity formalism, we carried out various investigations of the effects of chromaticity combined with beam-beam interaction on the luminosity. From weak-strong simulations, the effect of first-order chromaticity of alpha and beta functions and second-order tune chromaticity is almost invisible at the KEKB. On the contrary, results of strong-strong simulations show that large chromaticity of alpha and beta functions may cause remarkable loss of luminosity under the existence of dynamic effects due to beam-beam interaction. As an important result, we found that the chromatic $X-Y$ couplings are not small enough and can affect the luminosity at the present KEKB work point at around (44.515, 41.606). When chromaticity of one ring in ideal optics is considered in simulations, results of weak-strong simulations show that the luminosity degradation is around 5\%. Further results of strong-strong simulations show that the luminosity may decrease by around $10 \%$ and that the decrease depends slightly on bunch currents. Since we only considered chromaticity in one ring and did not consider machine errors, the $10 \%$ decrease should be considered as the minimum luminosity degradation.

Luminosity degradation caused by chromaticity depends greatly on betatron tunes. The chromaticity will enhance both the strength and the width of the synchrobeta resonance at $\nu_{x}-\nu_{y}+\nu_{z}=N$ because of the existence of nonzero chromatic $X-Y$ couplings. The present KEKB rings operate with tunes close to half integer and near resonance at $\nu_{x}-\nu_{y}+\nu_{z}=N$. The results of the present study provide an answer to the question of why chromatic $X-Y$ couplings can deteriorate the luminosity.

As compared to the successful knobs on chromaticities of alpha and beta functions at the IP, knobs on chromatic coupling are expected to be more powerful at KEKB. Since skew sextupoles can be used to control the chromatic $X-Y$ couplings, in the end of 2008, it was decided that skew sextupoles should be installed at both the HER and the LER. Tuning with skew sextupoles was commenced from May 2, 2009, at KEKB. Since then, beam operation at KEKB has been very successful and the chromatic $X-Y$ couplings at the IP have been reduced dramatically [18]. The corresponding luminosity gain was above $15 \%$ [22], and the peak luminosity exceeded $2 \times 10^{34} \mathrm{~cm}^{-2} \mathrm{~s}^{-1}$, which is twice the designed value.

\section{ACKNOWLEDGMENTS}

The authors would like to acknowledge the members of the KEKB commissioning group for careful beam measurements. Sincere thanks are due to Y. Cai (SLAC), E. Forest, M. Zobov (INFN), and Y. Funakoshi for help and discussions. We also appreciate valuable suggestions provided by K. Oide. This work is supported by the Large Scale Simulation Program No. 09/10-16(FY2009) of High Energy Accelerator Research Organization (KEK).

[1] KEKB B-factory Design Report No. KEK-Report-95-7, 1995.

[2] K. Ohmi et al., Phys. Rev. ST Accel. Beams 7, 104401 (2004).

[3] K. Ohmi et al., Proceedings of the 11th European Particle Accelerator Conference, Genoa, 2008 (EPS-AG, Genoa, Italy, 2008).

[4] K. Ohmi, in Proceedings of the 10th European Particle Accelerator Conference, Edinburgh, Scotland, 2006 (EPSAG, Edinburgh, Scotland, 2006).

[5] K. Ohmi, in Proceedings of the 40th ICFA Advanced Beam Dynamics Workshop on High Luminosity $e^{+} e^{-}$ Factories, Novosibirsk, Russia, 2008.

[6] A.B. Temnykh, in Proceedings of 13th International Conference on High Energy Accelerators, Novosibirsk, 1986, edited by A.N. Skrinsky (Novosibirsk Publishing House, Nauka, Siberian Division, 1987), Vol. 1, p. 78.

[7] M. Zobov, DAФNE Technical Note No. G-57, Frascati, 2001.

[8] A.B. Temnykh, in Proceedings of the 21st Particle Accelerator Conference, Knoxville, 2005 (IEEE, Piscataway, NJ, 2005).

[9] J.A. Crittenden, in Proceedings of the 10th European Particle Accelerator Conference, Edinburgh, Scotland, 2006 (EPS-AG, Edinburgh, Scotland, 2006).

[10] A. Valishev, in Proceedings of the 11th European Particle Accelerator Conference, Genoa, 2008 (Ref. [3]).

[11] Y. Seimiya and K. Ohmi, "Symplectic Expression for Chromaticity" (to be published). 
[12] For examples, see H. Zyngier, Report No. LAL 77/35, 1977; S. Peggs and G.F. Dell, in Proceedings of the Particle Accelerator Conference, Dallas, TX, 1995 (IEEE, New York, 1995); B.W. Montague, in Proceedings of the CERN Accelerator School, Oxford, England, 1985, edited by S. Turner (CERN, Geneva, 1987), p. 75.

[13] http://acc-physics.kek.jp/SAD/sad.html.

[14] D. Edwards and L. Teng, IEEE Trans. Nucl. Sci. 20, 885 (1973).

[15] K. Hirata, H. Moshammer, and F. Ruggiero, Part. Accel. 40, 205 (1993).

[16] K. Ohmi, Phys. Rev. E 62, 7287 (2000).
[17] A. Morita, H. Koiso, Y. Ohnishi, and K. Oide, Phys. Rev. ST Accel. Beams 10, 072801 (2007).

[18] Y. Ohnishi et al., Phys. Rev. ST Accel. Beams 12, 091002 (2009).

[19] K. Ohmi, K. Hirata, and K. Oide, Phys. Rev. E 49, 751 (1994).

[20] K. Ohmi, J. W. Flanagan, Y. Funakoshi, and K. Oide, Proceedings of the 23rd Particle Accelerator Conference, Vancouver, Canada, 2009.

[21] K. Oide and H. Koiso, Phys. Rev. E 49, 4474 (1994).

[22] Y. Funakoshi et al., Proceedings of the 23rd Particle Accelerator Conference, Vancouver, Canada, 2009. 